\title{
Transtorno do Espectro Autista (TEA) e hipersensibilidade alimentar: perfil nutricional e de sintomas gastrointestinais
}

\author{
Autistic Spectrum Disorder (ASD) and food \\ hypersensibility: nutritional profile and gastrointestinal \\ symptoms
}

Laura Moreira Goularte', Lilia Schug de Moraes', Eduarda de Souza Silva', Helayne Aparecida Maieves ${ }^{2}$, Lúcia Rota Borges ${ }^{3}$, Anne y Castro Marques ${ }^{4}$, Renata Torres Abib Bertacco ${ }^{5}$

'Acadêmicas do curso de Nutrição pela Universidade Federal de Pelotas - Campus Anglo, Pelotas, Rio Grande do Sul, Brasil.

${ }^{2}$ Docente do Departamento de Nutricão pela Universidade Federal de Pelotas. Doutora em Tecnologia de Alimentos pela Universidade Federal do Paraná - Curitiba, Paraná, Brasil.

${ }^{3}$ Docente do Departamento de Nutrição pela Universidade Federal de Pelotas. Doutora em Ciência e Tecnologia de Alimentos pela Universidade Federal de Pelotas - Pelotas, Rio Grande do Sul, Brasil.

${ }^{4}$ Docente do Departamento de Nutrição pela Universidade Federal de Pelotas. Doutora em Alimentos e Nutrição pela Universidade Estadual de Campinas - Campinas, São Paulo, Brasil.

${ }^{5}$ Docente do Departamento de Nutrição pela Universidade Federal de Pelotas. Doutora em Ciências Biológicas pela Universidade Federal do Rio Grande do Sul - Porto Alegre, Rio Grande do Sul, Brasil

Contato: Laura Moreira Goularte - lauragoularte99@gmail.com

\section{Resumo}

Objetivo: caracterizar o perfil nutricional e identificar a ocorrência de sintomas gastrointestinais na presença de hipersensibilidade alimentar, de crianças e adolescentes atendidos em um centro de referência no sul do Brasil. Método: estudo transversal realizado por meio da aplicação de questionário em um centro educacional para portadores do transtorno do espectro autista, na cidade de Pelotas, RS. Foram coletados dados sociodemográficos, bem como dados antropométricos e informações sobre dietas de exclusão, acompanhamento nutricional e sintomas gastrointestinais dos participantes. Resultados: Participaram do estudo 12 indivíduos, dos quais a maioria era do sexo masculino (75\%), criança $(91,6 \%)$ e da cor branca $(91,6 \%)$. Metade dos participantes estava com excesso de peso (25\% sobrepeso e $25 \%$ obesidade), além daqueles que já apresentavam risco de sobrepeso (16,7\%), enquanto somente $33,3 \%$ estavam eutróficos. Dentre as dietas de exclusão, as mais prevalentes foram lactose (75\%), caseína (25\%) e glúten (25\%), sendo que a maioria (75\%) dos alunos não fazia acompanhamento nutricional. Avaliando presença de sintomas gastrointestinais, flatulência (33,3\%) foi o mais relatado. Conclusão: destaca-se a importância de um suporte nutricional adequado para este público, uma vez que a maioria da amostra está com risco ou excesso de peso, além da presença de hipersensibilidade alimentar. Deve-se ter atenção no manejo de dietas de exclusão e estratégias para lidar com os sintomas gastrointestinais presentes, uma vez que sua eficácia ainda não possui comprovação científica para todos os casos. 
Palavras-chave: Transtorno do Espectro Autista. Intolerância alimentar. Estado nutricional. Gastroenteropatias.

\begin{abstract}
Objective: to characterize the nutritional profile and to identify the occurrence of gastrointestinal symptoms in the presence of food hypersensibility, of children and adolescents attended at a referral center in southern Brazill. Method: Crosssectional study conducted through the application of a questionnaire in an educational center for patients with Autistic spectrum disorder in Pelotas city, RS, Brazil. Socio-demographic data anthropometric data, information on exclusion diets, nutritional follow-up and gastrointestinal symptoms of the participants were colleted. Results: Participated in these study 12 individuals, of whom the majority were male (75\%), children (91, 6\%), and white color (91,6\%). Half of the participants were overweight (25\% overweight and 25\% obese), in addition to those who already presented risk of overweight (16, 7\%), while only 33,3\% were eutrophic. About the exclusion diets, the most prevalent were lactose (75\%), casein (25\%) and gluten (25\%), and most students (75\%) did not goy nutritional follow-up. Evaluating the presence of gastrointestinal symptoms, flatulence $(33,3 \%)$ was the most reported. Conclusion: The importance of a nutritional support is highlighted for this public, since most are at risk or overweight, in addition to the presence of allergies and food intolerances. Attention should be paid to the management of exclusion diets and strategies to deal with the gastrointestinal symptoms present, since its efficacy does not yet have scientific evidence for all cases.
\end{abstract}

Keywords: Autism Spectrum Disorder. Food intolerance. Nutritional status. Gastrointestinal diseases.

\title{
INTRODUÇÃO
}

O Transtorno do Espectro Autista (TEA) é sinalizado a partir de atrasos precoces no desenvolvimento de habilidades cognitivas e de comunicação, bem como nas interações sociais, resultando em importante dificuldade de adaptação ao longo da vida ${ }^{1}$. Estudos apontam que este transtorno ocorre predominantemente em indivíduos do sexo masculino, com uma prevalência em torno de 4 meninos para 1 menina ${ }^{2,3,4}$.

O TEA é uma desordem que apresenta enorme variabilidade de manifestações clínicas, podendo se relacionar a diversos tipos de interações entre os genes, mas também com fatores epigenéticos e exposição a fatores ambientais, o que torna um desafio o seu diagnóstico 5 .

Em relação a alterações físicas, estudos apontam que indivíduos com TEA podem apresentar desordens no trato gastrointestinal, tais como produção de enzimas digestivas diminuída, permeabilidade intestinal alterada e inflamações da parede intestinal. Essas alterações podem explicar a ocorrência de problemas absortivos, 
alergias e intolerâncias alimentares, assim como de sintomas comuns nesse público, tais como constipação crônica, flatulência, diarreia e dor abdominal ${ }^{6,7,8,9}$.

Na busca de melhor compreender as alterações relacionadas ao trato gastrointestinal, estudos têm investigado os hábitos alimentares de portadores de TEA, assim como a utilização de dietas especiais. Além da melhora dos sintomas gastrointestinais, algumas dietas de exclusão têm sido relacionadas com a redução de sintomas comportamentais. Neste cenário, as dietas que excluem glúten e caseína são as mais utilizadas, apesar de sua aplicação ser controversa e com evidências limitadas ${ }^{10,11}$.

Outros desafios relacionados à alimentação de indivíduos com transtorno do espectro autista são a seletividade alimentar e a preferência por alimentos ricos em carboidratos simples, que associados ao uso de alguns medicamentos, podem oferecer maior risco de sobrepeso e obesidade ${ }^{12}$. Além disso, o excesso de peso em indivíduos autistas pode ser um tanto mais difícil de evitar e reverter em comparação com indivíduos de desenvolvimento típico, uma vez que estes podem apresentar além da seletividade alimentar, dificuldade em realizar exercícios físicos e isolamento social, que colaboram para o sedentarismo ${ }^{13}$.

Considerando o exposto, o objetivo deste estudo foi caracterizar o perfil nutricional e identificar a ocorrência de sintomas gastrointestinais, na presença de hipersensibilidade alimentar, de crianças e adolescentes atendidos em um centro de referência no sul do Brasil.

\section{MÉTODO}

Tratou-se de um estudo transversal, realizado em um centro educacional para portadores do transtorno do espectro autista, localizado na cidade de Pelotas, RS, Brasil. A pesquisa foi realizada entre julho de 2015 e outubro de 2018, com dados coletados por acadêmicas do Curso de Nutrição da Universidade Federal de Pelotas (UFPel). Esta pesquisa compreende um recorte de um projeto maior, previamente aprovado pelo Comitê de Ética em Pesquisa da Faculdade de Medicina da UFPel, sob o número: 1.130.227.

Participaram deste estudo crianças e adolescentes cujos pais relataram diagnóstico médico de alguma alergia e/ou intolerância alimentar. Dos 325 indivíduos com TEA elegíveis para o estudo, 15 foram selecionados. Foram excluídos da amostra aqueles que não faziam nenhuma dieta de exclusão $(n=3)$. Os responsáveis legais assinaram o Termo de Consentimento Livre e Esclarecido, conforme estabelecido pela Resolução 466/12 do Conselho Nacional de Saúde ${ }^{14}$. 
As informações referentes a dados sociodemográficos (sexo, idade e cor da pele), à saúde e à dieta foram fornecidas pelo responsável que estava acompanhando o estudante, por meio de um questionário próprio.

Foram coletados os dados antropométricos peso e estatura, seguindo métodos preconizados pelo Sistema de Vigilância Alimentar e Nutricional15. A partir dessas informações, calculou-se o índice de massa corpórea (IMC) dos indivíduos, podendo assim classificar o estado nutricional dos mesmos conforme sexo e idade ${ }^{16,17}$.

Quanto à presença de alergia ou intolerância alimentar, foi feito o seguinte questionamento: "A criança/adolescente possui alguma dieta de exclusão?", tendo como opções de resposta: a) não possui; b) glúten; c) caseína; d) lactose; e) leite de vaca; f) outros. A partir disso, foi investigado se o uso da dieta de exclusão era devido à diagnóstico médico, e foi considerado então o diagnóstico referido no momento da entrevista (sem acesso a prontuário médico). Os responsáveis também informaram se a criança/adolescente fazia ou não algum acompanhamento nutricional.

Em relação aos sintomas gastrointestinais, foi investigado com que frequência a criança ou adolescente apresentava os seguintes sintomas: flatulência, constipação, diarreia aguda, diarreia crônica e dor abdominal, sendo possível responder nunca, raramente ou frequentemente.

Os dados foram categorizados da seguinte forma: sexo como feminino e masculino; idade como criança (o - 10 anos) e adolescente (10 - 19 anos); cor da pele como branca ou não branca; estado nutricional como magreza acentuada, magreza, eutrofia, risco de sobrepeso, sobrepeso, obesidade e obesidade grave; sintomas em nunca, raramente e frequentemente. Os dados foram tabulados no programa

Microsoft Excel, sendo apresentados por meio de análise descritiva.

\section{RESULTADOS}

Dos 12 indivíduos que atenderam aos critérios de inclusão, 9 (75\%) eram do sexo masculino, 11 (91,6\%) eram crianças (média de idade de 5,9 anos), e 11 eram da cor branca (91,6\%). Salienta-se que na variável cor da pele houve uma perda.

Na Figura 1 são apresentados os dados referentes ao estado nutricional dos estudantes com TEA e alergia/intolerância alimentar. Observou-se que a maioria da amostra foi composta por indivíduos com risco ou excesso de peso, de acordo com o IMC para a idade. 
Laura Moreira Goularte, Lilia Schug de Moraes, Eduarda de Souza Silva, Helayne Aparecida Maieves, Lúcia Rota Borges, Anne y Castro Marques, Renata Torres Abib Bertacco | Transtorno do Espectro Autista (TEA) e hipersensibilidade alimentar: perfil nutricional e de sintomas gastrointestinais

Figura 1 - Estado nutricional de crianças e adolescentes $(n=12)$ com diagnóstico de TEA e hipersensibilidade alimentar referida. Pelotas, RS, Brasil. 2018

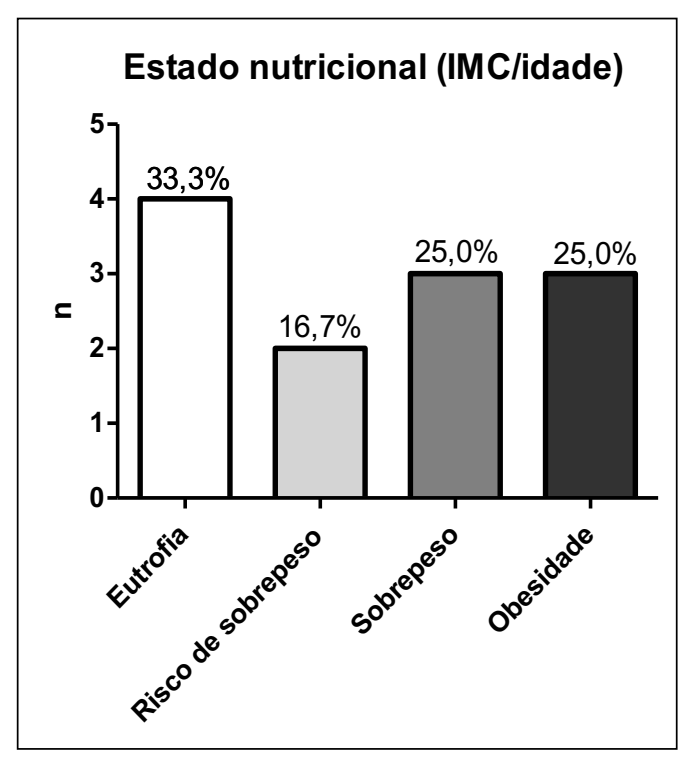

Na Tabela 1 são apresentados os dados referentes a dietas de exclusão, acompanhamento nutricional e sintomas gastrointestinais. É válido destacar que apenas um quarto da amostra fazia acompanhamento nutricional prévio ao momento da entrevista, dos quais um participante estava eutrófico, um com sobrepeso e um com obesidade (dados não mostrados).

Tabela 1 - Dieta de exclusão, acompanhamento nutricional e sintomas gastrointestinais de crianças e adolescentes (n = 12) com TEA e hipersensibilidade alimentar referida. Pelotas, RS, Brasil. 2018

\begin{tabular}{lll}
\hline Variável & N & $\%$ \\
\hline Dieta de exclusão & 3 & 25,0 \\
$\quad$ Glúten & 3 & 25,0 \\
Caseína & 9 & 75,0 \\
Lactose & 2 & 16,6 \\
Leite de vaca & 2 & 16,6 \\
Outros & & \\
Acompanhamento Nutricional & 3 & 25,0 \\
Sim & 3 & 75,0 \\
Não & 9 & \\
Flatulência & & 41,7 \\
Nunca & 5 & 25,0 \\
Raramente & 3 & 33,3 \\
Frequentemente & 4 & \\
Constipação & & 50,0 \\
Nunca & 6,3 \\
Raramente & 6 & 41,7 \\
Frequentemente & 5 & Continua
\end{tabular}


Laura Moreira Goularte, Lilia Schug de Moraes, Eduarda de Souza Silva, Helayne Aparecida Maieves, Lúcia Rota Borges, Anne y Castro Marques, Renata Torres Abib Bertacco | Transtorno do Espectro Autista (TEA) e hipersensibilidade alimentar: perfil nutricional e de sintomas gastrointestinais

\begin{tabular}{|c|c|c|}
\hline Variável & $\mathbf{N}$ & $\%$ \\
\hline \multicolumn{3}{|l|}{ Diarreia aguda } \\
\hline Nunca & 8 & 66,7 \\
\hline Raramente & 3 & 25,0 \\
\hline Frequentemente & 1 & 8,3 \\
\hline \multicolumn{3}{|l|}{ Diarreia crônica } \\
\hline Nunca & 12 & 100,0 \\
\hline \multicolumn{3}{|l|}{ Dor abdominal } \\
\hline Nunca & 7 & 58,3 \\
\hline Raramente & 5 & 41,7 \\
\hline \multicolumn{3}{|l|}{ Dieta de exclusão } \\
\hline Glúten & 3 & 25,0 \\
\hline Caseína & 3 & 25,0 \\
\hline Lactose & 9 & 75,0 \\
\hline Leite de vaca & 2 & 16,6 \\
\hline Outros & 2 & 16,6 \\
\hline \multicolumn{3}{|c|}{ Acompanhamento Nutricional } \\
\hline $\operatorname{Sim}$ & 3 & 25,0 \\
\hline Não & 9 & 75,0 \\
\hline \multicolumn{3}{|l|}{ Flatulência } \\
\hline Nunca & 5 & 41,7 \\
\hline Raramente & 3 & 25,0 \\
\hline Frequentemente & 4 & 33,3 \\
\hline \multicolumn{3}{|l|}{ Constipação } \\
\hline Nunca & 6 & 50,0 \\
\hline Raramente & 5 & 41,7 \\
\hline Frequentemente & 1 & 8,3 \\
\hline \multicolumn{3}{|l|}{ Diarreia aguda } \\
\hline Nunca & 8 & 66,7 \\
\hline Raramente & 3 & 25,0 \\
\hline Frequentemente & 1 & 8,3 \\
\hline \multicolumn{3}{|l|}{ Diarreia crônica } \\
\hline Nunca & 12 & 100,0 \\
\hline \multicolumn{3}{|l|}{ Dor abdominal } \\
\hline Nunca & 7 & 58,3 \\
\hline Raramente & 5 & 41,7 \\
\hline
\end{tabular}

\section{DISCUSSÃO}

No presente estudo, encontrou-se maioria de crianças e adolescentes do sexo masculino $(75,0 \%)$, resultado semelhante ao que foi encontrado por outros trabalhos ${ }^{2,18}$. Um estudo realizado por Jacquemont et al. ${ }^{4}$ em 2014, traz a possível justificativa para esta predominância do autismo em indivíduos do sexo masculino. O estudo investigou diferenças de bases moleculares entre meninos $(n=9206)$ e meninas ( $n=6379)$ no que se refere ao neurodesenvolvimento de uma deficiência, 
Laura Moreira Goularte, Lilia Schug de Moraes, Eduarda de Souza Silva, Helayne Aparecida Maieves, Lúcia Rota Borges, Anne y Castro Marques, Renata Torres Abib Bertacco | Transtorno do Espectro Autista (TEA) e hipersensibilidade alimentar: perfil nutricional e de sintomas gastrointestinais

mostrando que as meninas diagnosticadas com alguma alteração no desenvolvimento neurológico ou com TEA tiveram um número significativamente maior de mutações. No entanto, embora os dados mostrem um certo "modelo protetor" no sexo feminino, é necessário que se faça mais replicações deste estudo para confirmar o que foi concluído.

A média de idade encontrada foi de 5,9 \pm 2,37 anos e nenhuma criança participante do estudo possui idade menor que 3 anos. Este fato pode ser justificado pela dificuldade de acesso a um serviço especializado e possivelmente reflexo de um diagnóstico tardio ${ }^{19}$.

Em relação à presença de excesso de peso, o presente estudo obteve 50\%, além dos $16,7 \%$ de indivíduos que já apresentavam risco de sobrepeso. Estes resultados foram superiores ao obtido pelo Sistema de Vigilância Alimentar e Nutricional, no qual foi encontrada na população brasileira infanto-juvenil típica uma prevalência entre $29,26 \%$ e $31,74 \%$ de excesso de peso, de acordo com o índice IMC para Idade ${ }^{20}$. Em um estudo realizado no Ceará por Caetano et al. ${ }^{18}$ avaliando o perfil nutricional de 26 crianças com TEA, foi encontrada uma prevalência de 38,5\% de excesso de peso, também inferior ao resultado obtido no presente estudo. Em relação ao risco de sobrepeso, o estudo anterior ${ }^{18}$ obteve uma prevalência maior $(38,5 \%)$ que o estudo atual $(16,7 \%)$. Uma das possíveis justificativas ao cenário de excesso de peso encontrado repetidas vezes pode ser devido às dificuldades que estes indivíduos têm na alimentação, já que muitos deles possuem seletividade alimentar e sintomas gastrointestinais. Tais dificuldades podem levar tanto à carência de nutrientes essenciais quanto à repetição de uma rotina alimentar, sendo muitas vezes caracterizada pelo consumo aumentado de carboidratos refinados, o que favorece ainda mais o excesso de peso $0^{1,7,9}$.

Diante da presença de alergias e/ou intolerâncias alimentares, muitas vezes é necessário que haja restrição de determinado alimento, buscando melhora da qualidade de vida e dos sintomas que este consumo pode acarretar. Tratando-se da utilização de dietas isentas de glúten e/ou caseína entre portadores de TEA, a maioria dos estudos dá ênfase à influência de tais dietas como uma estratégia para amenizar e/ou melhorar certos comportamentos, o que não foi o foco do presente estudo. Apesar disso, alguns deles demonstram diminuição e melhora de sintomas gastrointestinais $^{21,22}$. Pode-se observar, entretanto, que grande parte dos estudos que buscaram investigar os possíveis efeitos das dietas que excluem glúten e/ou caseína 
não tiveram evidências suficientes para comprovar sua eficácia23,24. Uma revisão realizada por Lange et al. ${ }^{10}$ concluiu que, apesar da popularidade de tais intervenções nutricionais excluindo glúten e caseína, vários dos estudos realizados tiveram falhas metodológicas, e por isso não puderam trazer conclusões concretas sobre o tema. Sobre a exclusão de lactose da dieta de indivíduos com TEA, não foram encontrados artigos que pudessem comprovar efeitos positivos, apenas especulações e relatos. Sendo assim, se faz necessário um maior aprofundamento no assunto, por meio de investigações mais amplas acerca desta temática. Diversos estudos apontam a necessidade de acompanhamento nutricional para indivíduos com TEA. No que se refere à realização de dietas de exclusão, o acompanhamento nutricional é ainda mais importante para evitar a ocorrência de deficiências nutricionais nestes indivíduos ${ }^{26,27}$. Entretanto, com base no que foi exposto, é importante destacar que ainda não há evidências científicas suficientes para apoiar o uso de dietas de exclusão em pacientes com TEA, ainda que haja acompanhamento nutricional ${ }^{28}$.

Em relação aos sintomas gastrointestinais, um estudo brasileiro investigou os sintomas gastrointestinais em crianças e adolescentes com TEA (n=108), encontrando uma maior prevalência de flatulência entre os participantes ${ }^{25}$, dado que reforça o resultado obtido no presente estudo, em que a flatulência foi também a manifestação mais frequente. É válido ressaltar que a maioria dos artigos acerca desse tema são de revisão, esclarecendo de forma geral os sintomas mais recorrentes e possíveis motivos, mas sem avaliar a presença de sintomas gastrointestinais na população alvo ${ }^{6,29}$.

Destaca-se como limitação deste estudo o diagnóstico de alergia ou intolerância alimentar relatado, e não a partir de prontuário médico, além da incerteza quanto ao entendimento dos entrevistados quando questionados sobre dietas de exclusão. Apesar disso, os resultados apresentados são importantes, pois permitem obter um panorama do estado nutricional deste público tão particular. Ainda, de acordo com o exposto, é imprescindível que se façam maiores investigações a respeito do consumo alimentar e dos principais sintomas que acometem estes indivíduos, para que assim sejam elaboradas estratégias de intervenção comprovadamente eficazes.

\section{CONCLUSÃO}

Os participantes do estudo eram em sua maioria do sexo masculino e apresentaram excesso de peso. Neste cenário, o fato de uma minoria fazer acompanhamento nutricional é preocupante, uma vez que, agregado ao excesso de tecido adiposo, 
Laura Moreira Goularte, Lilia Schug de Moraes, Eduarda de Souza Silva, Helayne Aparecida Maieves, Lúcia Rota Borges, Anne y Castro Marques, Renata Torres Abib Bertacco | Transtorno do Espectro Autista (TEA) e hipersensibilidade alimentar: perfil nutricional e de sintomas gastrointestinais

estes indivíduos também apresentam alergia ou intolerância alimentar, necessitando de um suporte nutricional adequado.

Acerca das dietas de exclusão, destacou-se a restrição de lactose, e como principal sintoma a flatulência. Não foram encontradas na literatura evidências suficientes para apoiar o uso seguro das dietas de exclusão. Nesse sentido, é fundamental que haja mais investigações que resultem em orientações nutricionais, visando o manejo dos sintomas gastrointestinais, sem restrições desnecessárias. Destaca-se que não é indicada a realização de nenhum tipo de restrição alimentar sem a supervisão de um profissional nutricionista.

Ressalta-se ainda, que o excesso de peso é um fator de risco para a saúde em geral, e que a presença de sintomas gastrointestinais e hipersensibilidade alimentar pode reduzir significativamente a qualidade de vida do indivíduo. Portanto, é necessária uma atenção especial ao consumo alimentar deste público.

\section{AGRADECIMENTO}

Os autores agradecem às crianças/adolescentes e seus responsáveis pela participação, e ao Centro de atendimento ao Autista pela permissão para realização da coleta de dados.

\section{REFERÊNCIAS}

1. Carvalho JA, Santos CSS, Carvalho MP, Souza LS. Nutrição e Autismo: Considerações sobre a alimentação do autista. Revista Científica do ITPAC. 2012;5(1)Pub. 1.

2. Christensen DL, Baio J, Brau KVN, Bilder D, Charles J, Constantino JN, Daniels J, Durkin MS, Fitzgerald RT, Kurzius-Spencer M, Lee LC, Pettygrove S, Robinson C, Schulz E, Wells C, Wingate MS, Zahorodny W, Yeargin-Allsopp M. Prevalence and Characteristics of Autism Spectrum Disorder Among Children Aged 8 Years - Autism and Developmental Disabilities Monitoring Network, 11 Sites, United States, 2012. Surveillance Summaries. 2016;65(3):1-23.

3. Caetano, D. Classificação de transtornos mentais e de comportamento da CID10: descrições clínicas e diretrizes diagnósticas. In: Classificação de transtornos mentais e de comportamento da CID-10: descrições clínicas e diretrizes diagnósticas. Editora Artes Médicas Sul; 1993.

4. Jacquemont S, Coe BP, Hersch M, Duyzend MH, Krumm N, Bergmann S, Beckmann JS, Rosenfeld JA, Eichler EE. A higher mutational burden in females supports a "female protective model" in neurodevelopmental disorders. The American Journal of Human Genetics. 2014;94(3):415-425. 
Laura Moreira Goularte, Lilia Schug de Moraes, Eduarda de Souza Silva, Helayne Aparecida Maieves, Lúcia Rota Borges, Anne y Castro Marques, Renata Torres Abib Bertacco | Transtorno do Espectro Autista (TEA) e hipersensibilidade alimentar: perfil nutricional e de sintomas gastrointestinais

5. Willsey J, State MW. Autism spectrum disorder: from genes to neurobiology. Current Opinion in Neurobiology. 2015;30:92-99.

6. Hsiao EY. Gastrointestinal issues in autism spectrum disorder. Harvard Review of Psychiatry. 2014;22(2):104-111.

7. Leal M, Nagata M, Cunha NM, Pavanello U, Ferreira NVR. Terapia nutricional em crianças com transtorno do espectro autista. Cadernos da Escola de Saúde. 2015;1(13):1-13.

8. Fernandes MA, Vasconcelos MMF, Santos MPSS, Lima RMT, Veloso JO, Fernandes RF. Comportamento alimentar de crianças e adolescentes autistas atendidas em um centro integrado de educação especial. Revista de Enfermagem da UFPI. 2016;5(1):101-4.

9. Meguid NA, Anwar M, Bjorklund G, Hashish A, Chirumbolo S, Maha H, Sultan E. Dietary adequacy of Egyptian children with autism spectrum disorder compared to healthy developing children. Metabolic Brain Disease. 2017;32:607-615.

10. Lange KW, Hauser J, Reissmann A. Gluten-free and casein-free diets in the therapy of autism. Current Opinion in Clinical Nutrition \& Metabolic Care. 2015;18(6):572-575.

11. Alergia alimentar e Transtorno do espectro autista: existe relação?. Sociedade Brasileira de Pediatria (SBP) - Departamento Científico de Alergia. 2017. Disponível em: http://soperj.org.br/novo/imageBank/Alergia-alimentar-eTranstorno-2017.pdf. Acesso em: 12 out. 2018.

12. Kummer A, Barbosa IG, Rodrigues DH, Rocha NP, Rafael MS, Pfeilsticker L, Silva ACS, Teixeira AL. Frequência de sobrepeso e obesidade em crianças e adolescentes com autismo e transtorno do déficit de atenção/hiperatividade. Revista Paulista de Pedriatria. 2016;34(1):71-77.

13. Meguid NA, Kandeel WA, Wakeed KE, El-Nofely AA. Anthropometric assessment of a Middle Eastern group of autistic children. World Journal of Pediatrics. 2014;10(4):318-323.

14. Brasil. Ministério da Saúde. Conselho Nacional de Saúde. Comissão Nacional de ética em Pesquisa. Resolução 466/12. Dispõe sobre as diretrizes e normas regulamentadoras de pesquisas envolvendseres humanos. Brasília: Ministério da Saúde, 2012. Disponível em:

http://conselho.saude.gov.br/resolucoes/2012/Reso466.pdf. Acesso em: 30 out. 2014

15. Ministério da saúde. Orientações para a coleta e análise de dados antropométricos em serviços de saúde: Norma Técnica do Sistema de Vigilância Alimentar e Nutricional - SISVAN, Ministério da Saúde, Secretaria de Atenção à Saúde, Departamento de Atenção Básica. - Brasília: Ministério da Saúde, 2011. Acessado em 29 ago. 2018. Online. Disponível em:

http://bvsms.saude.gov.br/bvs/publicacoes/orientacoes_coleta_analise_dados _antro pometricos.pdf

16. Organização Mundial da Saúde - OMS. Growth reference data for 0-5 years, 2006. Disponível em:

http://www.who.int/childgrowth/standards/bmi_for_age/en/. Acesso em 30 ago. 2018.

17. Organização Mundial da Saúde - OMS. Growth reference data for 5-19 years, 2007. Disponível em: 
Laura Moreira Goularte, Lilia Schug de Moraes, Eduarda de Souza Silva, Helayne Aparecida Maieves, Lúcia Rota Borges, Anne y Castro Marques, Renata Torres Abib Bertacco | Transtorno do Espectro Autista (TEA) e hipersensibilidade alimentar: perfil nutricional e de sintomas gastrointestinais

http://www.who.int/growthref/who2007_bmi_for_age/en/. Acesso em 30 ago. 2018.

18. Caetano MV, Gurgel DC. Perfil nutricional de crianças portadoras do Transtorno do Espectro Autista. Revista Brasileira Promoção da Saúde. 2018;31(1):1-11.

19. Magalhães LS, Pereira ASP. Transtorno do espetro do autismo - Preocupações e apoios de famílias. Revista Educação Especial em Discussão. 2017;2(3):29-43.

20. Estado Nutricional de crianças e adolescentes do Brasil - Sistema de Vigilância Alimentar e Nutricional. Relatórios de Acesso Público. 2018. Disponível em: http://dabsistemas.saude.gov.br/sistemas/sisvanV2/relatoriopublico/index. Acesso em: 25 out. 2018.

21. Audisio A, Laguzzi J, Lavanda I, Leal M, Herrera J, Carrazana C, Cilento Pintos, CA. Mejora de los síntomas del autismo y evaluación alimentaria nutricional luego de la realización de una dieta libre de gluten y caseína en un grupo de niños con autismo que acuden a una fundación. Nutr Clín Diet Hosp. 2013;33(3):39-47.

22. Winburn E, Charlton J, McConachie H, McColl E, Parr J, O'Hare A, Baird G, Gringras P, Wilson DC, Adamson A, Adams S, Couteur AL. Parents' and child health professionals' attitudes towards dietary interventions for children with autism spectrum disorders. Journal of Autism Developmental Disorders. 2014;44(4):747-757.

23. Harris C, Card B. A pilot study to evaluate nutritional influences on gastrointestinal symptoms and behaviorpatterns in children with Autism Spectrum Disorder. Complement Ther Med. 2012; 20(6): 437-440.

24. Hyman SL, Stewart PA, Foley J, Peck R, Morris DD, Wang H, et al. The GlutenFree/ Casein-Free Diet : A Double-Blind Challenge Trial in Children with Autism. J Autism Dev Disord. 2016; 46(1): 205-220.

25. Pinho MA, Andrade M. Manifestações gastrointestinais em crianças com transtorno do espectro autista. Salvador: Universidade Federal da Bahia - 2015.

26. Magaganin T. Aspectos alimentares e nutricionais de crianças e adolescentes com Transtorno do Espectro Autista. Criciúma: Universidade do Extremo Sul Catarinense - 2019 .

27. Silva DV, Santos PNM, Silva DAV. Excesso de peso e sintomas gastrintestinais em um grupo de crianças autistas. Revista Paulista de Pediatria. 2020; 38:e2019080.

28. Dias EC, Rocha JS, Ferreira GB, Pena GG. Dieta exenta de gluten y caseína em el transtorno del espectro autista: una revisión sistemática. Revista Cuidarte. 2018;9(1):259-273.

29. Coury DL, Ashwood P, Fasano A, Fuchs, G, Geraghty, M, Kaul A, Mawe G, Patterson P, Jones NE. Gastrointestinal conditions in children with autism spectrum disorder: developing a research agenda. Pediatrics. 2012;130 suppl 2:S160-168.

Submissão: 13/03/2019

Aprovação: 02/07/2020 